\title{
LEUCOGRAMA, PROTEÍNA C REATIVA, ALFA-1 GLICOPROTEÍNA ÁCIDA E VELOCIDADE DE HEMOSSEDIMENTAÇÃO NA APENDICITE AGUDA
}

\author{
Bruno Ramalho de CARVALHO ${ }^{1}$, Augusto DIOGO-FILHO ${ }^{2}$, \\ Cleiton FERNANDES ${ }^{1}$ e Cristiane Borges BARRA ${ }^{1}$
}

RESUMO - Racional - O diagnóstico da apendicite aguda é clínico, mas alguns indivíduos podem apresentar sinais e sintomas pouco característicos. As dificuldades diagnósticas ainda conduzem os cirurgiões à realização de laparotomias desnecessárias, que atingem índices de $15 \%$ a $40 \%$. Os exames laboratoriais, assim, podem se tornar complementos úteis no diagnóstico da apendicite aguda. O leucograma parece ser o de maior valor, mas a dosagem das proteínas de fase aguda, em especial da proteína C reativa, é objeto de vários estudos. Casuística e Método - Estudo longitudinal prospectivo, envolvendo 63 pacientes submetidos a apendicectomia por suspeita de apendicite aguda no Hospital das Clínicas da Universidade Federal de Uberlândia, MG, de cujas amostras de sangue foram feitas dosagens das proteínas de fase aguda e leucograma. Resultados - Foram 44 pacientes do sexo masculino e a faixa etária predominante entre 11 e 30 anos. A apendicite aguda foi mais freqüente no sexo masculino $(69,8 \%)$ e na faixa etária entre 11 e 30 anos. O tipo flegmonoso foi mais freqüente $(52,4 \%)$. O leucograma mostrou-se aumentado em $74,6 \%$ dos casos e a elevação da proteína $\mathrm{C}$ reativa foi observada em $88,9 \%$. As dosagens de alfa-1 glicoproteína ácida e velocidade de hemossedimentação foram predominantemente normais. A proteína $\mathrm{C}$ reativa esteve aumentada em mais de $80 \%$ dos casos em todas as idades. O leucograma e a proteína C reativa alteraram-se em $80 \%$ dos pacientes com até 24 horas do início dos sintomas. Com tempo de evolução dos sintomas superior a 24 horas, o leucograma mostrou-se alterado em $69,7 \%$ dos casos e a proteína C reativa, em $97 \%$. Sensibilidade e especificidade do leucograma foram de $88,7 \%$ e $20 \%$. Para a proteína C reativa, foram de $88,9 \%$ e $10 \%$, respectivamente. A dosagem da proteína C reativa apresentou maior sensibilidade $(96,9 \%)$ para os casos de evolução com mais de 24 horas, sem qualquer especificidade, entretanto, alfa-1 glicoproteína ácida e velocidade de hemossedimentação mostraram-se pouco sensíveis e específicos. Conclusões - O leucograma e a proteína C reativa apresentam-se alterados de forma significativa nos casos de apendicite aguda, independentemente do sexo ou da faixa etária. O leucograma e, principalmente, a proteína $\mathrm{C}$ reativa devem ser exames considerados em indivíduos com tempo de evolução sintomática superior a 24 horas. Valores aumentados, entretanto, devem ser somados e não substituir a avaliação clínica do médico examinador. Dosagens de velocidade de hemossedimentação e da alfa-1 glicoproteína ácida não trazem auxílio ao diagnóstico da apendicite aguda.

DESCRITORES - Apendicite. Abdome agudo. Dor abdominal. Proteínas de fase aguda. Proteína C.

\section{INTRODUÇÃO}

A apendicite aguda responde por aproximadamente $4 \%$ das dores abdominais atendidas em serviços de pronto-socorro e é a mais comum dentre as que necessitam de tratamento cirúrgico ${ }^{(11)}$. GRAFFEO e COUNSELMAN ${ }^{(11)}$ mostram incidência anual de 23 casos para cada 10.000 indivíduos entre 10 e 20 anos de idade.

O diagnóstico da apendicite aguda é clínico, com base na história da moléstia e nos achados ao exame físico ${ }^{(16,26)}$. Alguns indivíduos, entretanto, podem apresentar sinais e sintomas pouco característicos e as dificuldades diagnósticas ainda conduzem os cirurgiões à realização de laparotomias desnecessárias, que atingem índices de $15 \%$ a $40 \%{ }^{(2,3)}$. OOSTERHUIS et al. ${ }^{(19)}$, chegam a mencionar ocorrência de $75 \%$. A morbidade secundária às laparotomias desnecessárias é mencionada por HALLAN e ASBERG $^{(15)}$, que apontam sua importante repercussão econômica e social, uma vez que a afecção atinge principalmente a faixa etária economicamente ativa (adultos jovens, em sua maioria).

Departamento de Cirurgia da Faculdade de Medicina da Universidade Federal de Uberlândia, Hospital das Clínicas de Uberlândia, Minas Gerais

Faculdade de Medicina da Universidade Federal de Uberlândia; ${ }^{2}$ Departamento de Cirurgia da Faculdade de Medicina da Universidade Federal de Uberlândia, MG. Endereço para correspondência - Dr. Bruno Ramalho de Carvalho - Av. Caramuru, 2100 - apt. 1.324 - Alto da Boa Vista - 14030-000 - Ribeirão Preto, SP.

e-mail: brunoramalho@hotmail.com / brunoramalho@bol.com.br 
A real importância dos testes laboratoriais complementares para o diagnóstico da apendicite permanece controversa ${ }^{(2)}$. Para os casos de difícil conclusão diagnóstica, os exames laboratoriais podem se tornar úteis, diminuindo, por conseguinte, a incidência de erros. Entre os exames disponíveis, o leucograma (LCG) parece ser o exame complementar de maior importância no auxílio ao diagnóstico da apendicite aguda ${ }^{(5)}$.

As proteínas de fase aguda são glicoproteínas liberadas na circulação em resposta a situações de estresse, como na vigência de processos inflamatórios agudos. Vários estudos têm investigado o real valor desses marcadores na complementação diagnóstica de quadros inflamatórios e infecciosos ${ }^{(6)}$. A proteína $\mathrm{C}$ reativa (PCR), em especial, é objeto de incontáveis artigos acerca do diagnóstico laboratorial complementar da apendicite aguda ${ }^{(3,7,8,13-15,21,22, ~ 24, ~ 27), ~ m a s ~ a i n d a ~}$ existem controvérsias sobre a sua verdadeira importância ${ }^{(25)}$. Estudos relativos à velocidade de hemossedimentação (VHS) e à alfa-1glicoproteína ácida (AGA) são escassos ${ }^{(10,17)}$.

O presente estudo, assim, propôs-se a avaliar a importância do LCG e das dosagens de PCR, AGA e VHS na complementação à abordagem dos casos de dor abdominal com suspeita de apendicite aguda.

\section{CASUÍSTICA}

A população do estudo foi composta por 73 pacientes submetidos a apendicectomia por suspeita diagnóstica de apendicite aguda, atendidos no pronto-socorro do Hospital de Clínicas da Universidade Federal de Uberlândia MG (HCUFU) entre junho e dezembro do ano de 2001. Foram avaliados 63 indivíduos com diagnóstico histológico de apendicite aguda confirmado pelo Serviço de Anatomia Patológica do HCUFU e 10 indivíduos cujo diagnóstico clínico não foi confirmado pela histologia.

\section{MÉTODO}

Estudo longitudinal prospectivo. Foram colhidas amostras de sangue dos pacientes, momentos antes da realização da cirurgia, para dosagem de PCR (valor de referência: $<5 \mathrm{mg} / \mathrm{L}$ ), AGA (valor de referência: de 0,5 a 1,3 g/L), VHS (valor de referência: até $20 \mathrm{~mm}$ ) e contagem de leucócitos (valor de referência: 11.000 células $/ \mathrm{mm}^{3}$, com até 700 bastonetes $/ \mathrm{mm}^{3}$ ). A obtenção dos quatro resultados dos referidos exames foi o critério de inclusão dos pacientes na amostra a ser estudada. Foram realizadas análises estatísticas multivariadas, para as quais se utilizaram testes de qui-quadrado, considerando significativo $P<0,05$.

\section{RESULTADOS}

A apendicite aguda foi mais freqüente no sexo masculino, com $69,8 \%$ dos casos $(P<0,05)$, e na faixa etária entre 11 e 30 anos, com $60,3 \%(P>0,05)$. Quanto ao tipo histológico, o flegmonoso foi mais freqüente $(52,4 \%)$, seguido pelo necrosante $(42,8 \%)$.

A contagem de leucócitos mostrou-se aumentada em 74,6\% dos casos (média de 14.100 células $/ \mathrm{mm}^{3}$ ), envolvendo $70,4 \%$ dos pacientes do sexo masculino e $84,2 \%$ do sexo feminino. As médias foram de 13.800 células $/ \mathrm{mm}^{3}$ e 14.900 células $/ \mathrm{mm}^{3}$, respectivamente. O desvio à esquerda foi observado em $50,8 \%$ dos casos, mais freqüentemente nos indivíduos do sexo masculino (Tabela 1).

A elevação dos níveis da PCR foi observada em $88,9 \%$ dos pacientes (média de 76,22 mg/L), sendo 86,4\% dos homens e 89,5\% das mulheres. Os respectivos valores médios foram de $82,45 \mathrm{mg} / \mathrm{L}$ e $61,8 \mathrm{mg} / \mathrm{L}$. AGA e VHS apresentaram-se inalteradas na maior parte dos casos (Tabela 1).

A PCR mostrou-se aumentada em mais de $80 \%$ dos casos em todas as faixas etárias estudadas, o que não ocorreu com o leucograma e as demais proteínas de fase aguda (Tabela 2).

Observou-se contagem de leucócitos elevada em $80 \%$ dos pacientes com até 24 horas do início dos sintomas, índice semelhante ao da PCR $(P<0,05)$. Para os indivíduos com tempo de evolução dos sintomas (TE) superior a 24 horas, entretanto, a PCR elevou-se em 97\% dos casos, superando o LCG, alterado em 69,7\% $(P<0,05)$.

As Tabelas 3 e 4 mostram os resultados dos exames laboratoriais de acordo com o TE e com tipo histológico encontrado.

TABELA 1 - Exames laboratoriais de acordo com o sexo (n) nos pacientes submetidos a apendicectomias com diagnóstico confirmado pelo exame histológico

\begin{tabular}{|c|c|c|c|c|c|c|c|c|c|c|}
\hline \multirow[t]{2}{*}{ Sexo } & \multicolumn{2}{|c|}{$\begin{array}{c}\text { LCG } \\
\text { (células/mm } / \mathbf{m m}^{3} \text { ) }\end{array}$} & \multicolumn{2}{|c|}{$\begin{array}{l}\text { Desvio à esquerda } \\
\left.\text { (células } / \mathbf{m m}^{3}\right)\end{array}$} & \multicolumn{2}{|c|}{$\begin{array}{c}\text { PCR } \\
(\mathrm{mg} / \mathrm{L})\end{array}$} & \multicolumn{2}{|c|}{$\begin{array}{r}\text { AGA } \\
(\mathrm{g} / \mathrm{L})\end{array}$} & \multicolumn{2}{|c|}{$\begin{array}{c}\text { VHS } \\
\left(\mathbf{m m}^{3}\right)\end{array}$} \\
\hline & $\leq 11.000$ & $>11.000$ & $\leq 700$ & $>700$ & $\leq 05$ & $>05$ & $\leq 1,3$ & $>1,3$ & $\leq 20$ & $>20$ \\
\hline (44) & (14) & (30) & (20) & (24) & (6) & (38) & (33) & (11) & (37) & (7) \\
\hline Feminino $^{[b]}$ & $15,8 \%$ & $84,2 \%$ & $57,9 \%$ & $42,1 \%$ & $10,5 \%$ & $89,5 \%$ & $73,7 \%$ & $26,3 \%$ & $68,4 \%$ & $31,6 \%$ \\
\hline (63) & (17) & (46) & (31) & (32) & (8) & (55) & (47) & (16) & (50) & (13) \\
\hline
\end{tabular}

Análise multivariada: [a] $P<0,05$; [b] $P<0,05$; [c] $P<0,05$ 
Carvalho BR, Diogo-Filho A, Fernandes C, Barra CB. Leucograma, proteína C reativa, alfa-1 glicoproteína ácida e velocidade de hemossedimentação na apendicite aguda

TABELA 2 - Exames laboratoriais de acordo com a faixa etária (n) nos pacientes submetidos a apendicectomias terapêuticas

\begin{tabular}{|c|c|c|c|c|c|c|c|c|c|c|}
\hline \multirow[t]{2}{*}{ Faixa etária } & \multicolumn{2}{|c|}{$\begin{array}{c}\text { LCG } \\
(\text { células/mm³) }\end{array}$} & \multicolumn{2}{|c|}{$\begin{array}{l}\text { Desvio à esquerda } \\
\left(\text { células } / \mathbf{m m}^{3}\right)\end{array}$} & \multicolumn{2}{|c|}{$\begin{array}{c}\text { PCR } \\
(\mathrm{mg} / \mathrm{L})\end{array}$} & \multicolumn{2}{|c|}{$\begin{array}{l}\text { AGA } \\
(g / L)\end{array}$} & \multicolumn{2}{|c|}{$\begin{array}{l}\text { VHS } \\
\left(\mathbf{m m}^{3}\right)\end{array}$} \\
\hline & $\leq 11.000$ & $>11.000$ & $\leq 700$ & $>700$ & $\leq 05$ & $>05$ & $\leq 1,3$ & $>1,3$ & $\leq 20$ & $>20$ \\
\hline (21) & (3) & (18) & (11) & (10) & (4) & (17) & (15) & (6) & (17) & (4) \\
\hline 21 a $30 \operatorname{anos}^{[b]}$ & $41,2 \%$ & $58,8 \%$ & $47,1 \%$ & $52,9 \%$ & 0 & $100 \%$ & $68,8 \%$ & $31,2 \%$ & $76,5 \%$ & $23,5 \%$ \\
\hline (14) & (1) & (13) & (7) & (7) & (1) & (13) & (10) & (4) & $(12)$ & $(2)$ \\
\hline$>40 \operatorname{anos}^{[\mathrm{d}]}$ & $36,5 \%$ & $63,5 \%$ & $45,5 \%$ & $54,5 \%$ & 0 & $100 \%$ & $90,9 \%$ & $9,1 \%$ & $72,7 \%$ & $27,3 \%$ \\
\hline (11) & (4) & (7) & $(5)$ & $(6)$ & $(0)$ & $(11)$ & $(10)$ & (1) & (8) & (3) \\
\hline
\end{tabular}

Análise multivariada: [a] $P<0,05$; [b] $P<0,05$; [c] $P<0,05$; [d] $P<0,05$

TABELA 3 - Exames laboratoriais de acordo com o tempo de evolução dos sintomas (n) nos pacientes submetidos a apendicectomias terapêuticas

\begin{tabular}{|c|c|c|c|c|c|c|c|c|c|c|}
\hline \multirow[t]{2}{*}{$\begin{array}{l}\text { Tempo de } \\
\text { evolução }\end{array}$} & \multicolumn{2}{|c|}{$\begin{array}{c}\text { LCG } \\
\text { (células/mm } / \mathbf{m m}^{3} \text { ) }\end{array}$} & \multicolumn{2}{|c|}{$\begin{array}{l}\text { Desvio à esquerda } \\
\left(\text { células } / \mathbf{m m}^{3}\right)\end{array}$} & \multicolumn{2}{|c|}{$\begin{array}{c}\text { PCR } \\
(\mathrm{mg} / \mathrm{L})\end{array}$} & \multicolumn{2}{|c|}{$\begin{array}{l}\text { AGA } \\
(\mathrm{g} / \mathrm{L})\end{array}$} & \multicolumn{2}{|c|}{$\begin{array}{l}\text { VHS } \\
\left(\mathbf{m m}^{3}\right)\end{array}$} \\
\hline & $\leq 11.000$ & $>11.000$ & $\leq 700$ & $>700$ & $\leq 05$ & $>05$ & $\leq 1,3$ & $>1,3$ & $\leq 20$ & $>20$ \\
\hline$(30)$ & (6) & (24) & (15) & (15) & (6) & (24) & (27) & (3) & (27) & (3) \\
\hline$>24 \operatorname{horas}^{[b]}$ & $30,3 \%$ & $69,7 \%$ & $51,5 \%$ & $48,5 \%$ & $3 \%$ & $97 \%$ & $66,7 \%$ & $33,3 \%$ & $72,7 \%$ & $27,3 \%$ \\
\hline
\end{tabular}

Análise multivariada: [a] $P<0,05$; [b] $P<0,05$

TABELA 4 - Exames laboratoriais de acordo com o tipo histológico da apendicite aguda (n)

\begin{tabular}{|c|c|c|c|c|c|c|c|c|c|c|}
\hline \multirow[t]{2}{*}{ Histologia } & \multicolumn{2}{|c|}{$\begin{array}{c}\text { LCG } \\
\left(\text { células/mm } / \mathbf{m m}^{3}\right)\end{array}$} & \multicolumn{2}{|c|}{$\begin{array}{l}\text { Desvio à esquerda } \\
\left(\text { células } / \mathbf{m m}^{3}\right)\end{array}$} & \multicolumn{2}{|c|}{$\begin{array}{c}\text { PCR } \\
(\mathbf{m g} / \mathbf{L})\end{array}$} & \multicolumn{2}{|c|}{$\begin{array}{l}\text { AGA } \\
(\mathrm{g} / \mathbf{L})\end{array}$} & \multicolumn{2}{|c|}{$\begin{array}{l}\text { VHS } \\
\left(\mathbf{m m}^{3}\right)\end{array}$} \\
\hline & $\leq 11.000$ & $>11.000$ & $\leq 700$ & $>700$ & $\leq 05$ & $>05$ & $\leq 1,3$ & $>1,3$ & $\leq 20$ & $>20$ \\
\hline (3) & (2) & (1) & (3) & $(0)$ & $(0)$ & (3) & (2) & (1) & (3) & $(0)$ \\
\hline Flegmonosa $^{[b]}$ & $24,3 \%$ & $75,7 \%$ & $48,5 \%$ & $51,5 \%$ & $12,1 \%$ & $87,9 \%$ & $78,8 \%$ & $21,2 \%$ & $84,9 \%$ & $15,1 \%$ \\
\hline (27) & (8) & (19) & (12) & $(15)$ & $(0)$ & $(27)$ & (19) & $(8)$ & (19) & $(8)$ \\
\hline
\end{tabular}

Análise multivariada: [a] $P=0,06$; [b] $P<0,05$; [c] $P<0,05$

O LCG apresentou sensibilidade de $88,7 \%$ e especificidade de $20 \%$, considerada toda a população do estudo. Com tempo de evolução dos sintomas de até 24 horas, entretanto, a sensibilidade foi de $80 \%$, com $16 \%$ de especificidade e valores preditivos positivo e negativo de $82,7 \%$ e $14,3 \%$, respectivamente.

A dosagem da PCR apresentou maior sensibilidade $(96,9 \%)$ para os casos de evolução com mais de 24 horas, sem qualquer especificidade, entretanto, e valores preditivos positivo e negativo de $88,9 \%$ e $0 \%$, respectivamente. AGA e VHS mostraram-se pouco sensíveis e específicos.

\section{DISCUSSÃO}

A alta incidência de laparotomias desnecessárias conduz-nos à reflexão acerca do papel dos exames complementares no auxílio ao 
diagnóstico da apendicite aguda. Com a intenção de se diminuir a adversidade do ato operatório desnecessário, a solicitação de exames complementares é proposta em inúmeros estudos, mas permanece controversa $^{(2)}$. FLUM et al. ${ }^{(9)}$ demonstraram que não houve diminuição significativa no número de apendicectomias desnecessárias no Hospital Washington State, entre os anos de 1987 e 1998, mesmo com a utilização de exames modernos como a tomografia computadorizada, a ultra-sonografia e a laparoscopia. Observaram, entretanto, diminuição do índice de laparotomias desnecessárias com a solicitação das proteínas de fase aguda além do LCG. A incidência de $21 \%$ (dado nãopublicado) passou a ser de $13,7 \%$.

Com todas as controvérsias em torno da avaliação dos casos suspeitos de apendicite aguda, faz-se necessária maior elucidação no que concerne aos mais variados exames disponíveis, sejam laboratoriais ou de imagem.

\section{O leucograma (LCG)}

O LCG encontra-se alterado em $70 \%$ a $90 \%$ dos pacientes com apendicite aguda, com sensibilidade e especificidade que chegam, respectivamente, a $92 \%$ e $100 \%{ }^{(23)}$. Vários autores preconizam sua realização, considerando-o teste de valor na complementação diagnóstica da afecção ${ }^{(5)}$. Há evidências de que o LCG seja o exame de maior sensibilidade quando realizado pouco tempo após o início do quadro doloroso, perdendo seu valor quando da existência de quadros de evolução prolongada e diagnóstico retardado ${ }^{(6)}$. GRÖNROOS e GRÖNROOS ${ }^{(13)}$, em análise retrospectiva, mencionam a importância do exame na apendicite não complicada e afirmam que níveis normais associados à PCR também normal excluem a possibilidade de apendicite. Isso é válido também para a população idosa ${ }^{(12)}$.

O presente estudo não demonstrou ser o LCG exame essencial para o diagnóstico da apendicite aguda, pois, apesar da sensibilidade de $88,7 \%$, mostrou-se pouco específico (20\%). Entretanto, elevação do número de leucócitos para os casos de apendicite é mais significativa que desvio à esquerda e, para indivíduos entre 11 e 20 anos, supera, também, a PCR de forma significativa.

\section{A proteína Creativa (PCR)}

A PCR apresenta sensibilidade e especificidade que chegam, respectivamente, a $87 \%$ e $82 \%$ na apendicite aguda, mas ambas atingem $90 \%$ em outros estudos ${ }^{(6,15)}$. Sua utilidade é mencionada principalmente após 6 a 12 horas do início dos sintomas ${ }^{(4,12)}$. DAVIES et al. ${ }^{(7)}$ colocaram a PCR como melhor indicador em relação ao LCG. Nos casos de perfuração do apêndice, parece ser ainda de maior valor ${ }^{(2,20)}$. Em contrapartida, HALLAN e ASBERG ${ }^{(15)}$ afirmaram que, apesar da necessidade de cautela na decisão de abordagem operatória com PCR normal, é exame inferior em importância em relação ao LCG.
A elevação da PCR aparece no presente estudo como alteração significativa quando comparada aos demais exames estudados, principalmente em indivíduos com mais de 21 anos e naqueles com mais de 24 horas de quadro sintomático. A sensibilidade é alta (96,9\%) nos casos com mais de 24 horas de quadro sintomático, apesar de inespecífica.

ALBU et al. ${ }^{(1)}$ acharam $100 \%$ de sensibilidade para valores superiores a $25 \mathrm{mg} / \mathrm{L}$ em pacientes com 12 ou mais horas de evolução sintomática e chegaram à conclusão de que valores inferiores a este após 12 horas são indicativos de resolução espontânea do quadro inflamatório. Na presente série, apenas $16,1 \%$ dos indivíduos apresentaram valor inferior a $25 \mathrm{mg} / \mathrm{L}$, tendo mais de 12 horas de evolução sintomática.

\section{A velocidade de hemossedimentação (VHS)}

Não se observa importância significativa da VHS nos casos de apendicite aguda. Apesar de ter importância na vigência de processos inflamatórios com duração superior a 24 horas, a VHS pode permanecer elevada após a resolução do quadro, além de sofrer a influência de alterações hematológicas, como anemia, policitemia e dimorfismos eritrocitários, idade e sexo ${ }^{(6,17)}$. Prefere-se, portanto, a dosagem da PCR e a contagem de leucócitos a este exame ${ }^{(19)}$. Ainda assim, PELTOLA et al. ${ }^{(21)}$ observaram que $51 \%$ dos pacientes com apendicite na faixa etária pediátrica, considerada até os 16 anos, apresentaram VHS aumentada após 24 horas do início dos sintomas. Não consideraram, também, a idade como fator de influência nas dosagens da VHS.

O presente estudo não observou relação entre o tempo de evolução dos sintomas e a elevação dos níveis da VHS. Não houve correlação entre sexo ou idade também. Os níveis se elevam após 24 horas de evolução, mas a maior parte dos pacientes permanece com valores dentro da normalidade. A VHS mostrou-se, ainda, exame de sensibilidade e especificidade baixas, o que nos conduz desconsiderála na propedêutica de casos suspeitos de apendicite aguda.

\section{A alfa-1 glicoproteína ácida (AGA)}

A AGA não tem função biológica bem definida, mas coloca-se no rol dos principais marcadores de atividade inflamatória ${ }^{(10)}$. Até agora, parece ser útil a dosagem da AGA no acompanhamento de pacientes após realização de quimioterapia, na avaliação de recurrências neoplásicas.

Não foram encontrados estudos avaliando a importância da AGA no diagnóstico da apendicite aguda, mas por ser referida como indicador alterado precocemente na presença de processos agressores ao organismo, mesmo antes do aparecimento de sintomas, acredita-se por bem investigar se ocorrem alterações nos casos suspeitos.

Os pacientes envolvidos neste estudo não apresentaram elevações significativamente importantes dos níveis da AGA, mas estas ocorreram 
com maior freqüência em relação ao aumento da VHS em ambos os sexos, principalmente abaixo dos 40 anos de idade e com mais de 24 horas de evolução sintomática. As baixas sensibilidade e especificidade, no entanto, não sugerem que sua dosagem elemento promissor no auxílio ao diagnóstico da apendicite aguda.

\section{CONCLUSÕES}

Alterações da PCR são significativamente mais freqüentes para os casos de apendicite aguda em relação ao LCG, à AGA e à VHS, principalmente para casos com tempo de evolução superior a 24 horas.

As dosagens da VHS e da AGA encontram-se alteradas em pequena quantidade de pacientes e não auxiliam no diagnóstico da apendicite aguda.

O LCG e a dosagem da PCR são exames de boa sensibilidade e, apesar da especificidade reduzida, devem ser considerados na propedêutica de todos os casos suspeitos de apendicite aguda. Valores aumentados, entretanto, devem ser somados e não substituir a avaliação clínica do médico examinador.

Carvalho BR, Diogo-Filho A, Fernandes C, Barra CB. Leucocyte count, C reactive protein, alpha-1 acid glycoprotein and erithrocyte sedimmentation rate in acute appendicitis. Arq Gastroenterol 2003;40(1):25-30

ABSTRACT - Background - The diagnosis of acute appendicitis is clinic, but in some cases, it can present unusual symptoms. The diagnostic difficulties still lead surgeons to unnecessary laparotomies, which reach rates from $15 \%$ to $40 \%$. Laboratory exams, then, may become important to complement appendicitis diagnosis. The leucocyte count seems to be the most important value, but measurement of acute phase proteins, specially, the C-reactive protein, is object of several studies. Patients and Methods - This was a prospective study, involving 63 patients submitted to appendecectomies for acute appendicitis suspicion, in "Hospital das Clínicas", Federal University of Uberlândia, MG, Brazil, in whose blood were made dosages of acute phase proteins and the leucocyte count. Results - The sample was composed by 44 male and 19 female patients, and the majority of them was between 11 and 30 years of age. The flegmonous type was the most freqüent (52.4\%). The leucocyte count was altered in $74.6 \%$ of the cases and C-reactive protein elevation was observed in $88.9 \%$. The alfa- 1 acid glycoprotein and the erithrocyte sedimmentation rate were predominantly normal. The C-reactive protein was augmented in more than $80 \%$ of the cases in all ages. Leucocyte count and C-reactive protein were altered in $80 \%$ of the patients with the limit of 24 hours from the beginning of symptoms. With clinical evolution time superior than 24 hours, the leucocyte count was altered in $69.7 \%$ of the cases, whereas C-reactive protein was in $97 \%$. Sensibility and specificity of the leucocyte count were $88.7 \%$ and $20 \%$. For the C-reactive protein, the values were, respectively, $88.9 \%$ and 10\%. C-reactive protein dosage presented more sensible in cases with more than 24 hours of evolution (96.9\%), although with no specificity. The alfa-1 acid glycoprotein and erithrocyte sedimmentation rate presented low sensitivity and specificity. Conclusion - The leucocyte count and the C-reactive protein present significantly altered in acute appendicitis cases, independent from genre or age interval. The leucocyte count and, mainly, the C-reactive protein must be considered in individuals with more than 24 hours of clinical evolution. Augmented values, as a matter of fact, should never substitute the doctor's clinical examination, but complement it. The erithrocyte sedimmentation rate and the alfa1 acid glycoprotein do not contribute to acute appendicitis diagnosis

HEADINGS - Appendicitis. Abdomen, acute. Abdominal pain. Acute-phase proteins. Protein C.

\section{REFERÊNCIAS BIBLIOGRÁFICAS}

1. Albu E, Miller BM, Choi Y, Lakhanpal S, Murthy RN, Gerst PH. Diagnostic value of C-reactive protein in acute appendicitis. Dis Colon Rectum 1994;37:4951.

2. Andersson RE, Hugander AP, Ghazi SH, Ravn H, Offenbartl SK, Nystrom PO, Olaison GP. Diagnostic value of disease history, clinical presentation, and inflammatory parameters of appendicitis. World J Surg 1999;23:133-40.

3. Asfar S, Safar H, Khoursheed M, Dashti H, al-Bader AJR. Would measurement of $\mathrm{C}$-reactive protein reduce the rate of negative exploration for acute appendicitis? J R Coll Surg Edinb 2000;45:21-4.

4. Brasileiro-Filho G, Pereira FEL, Pittella JEH, Bambirra EA, Barbosa AJA. Apêndice íleo-cecal. In: Brasileiro-Filho G, Pereira FEL, Pittella JEH, Bambirra EA, Barbosa AJA, editores. Bogliolo patologia. 5 ed. Rio de Janeiro: Guanabara Koogan; 1994. p.594.

5. Carvalho BR, Diogo-Filho A, Alfredo AS, Pagani AM, Fernandes C. Importância dos exames complementares no diagnóstico da apendicite aguda. Prat Hosp $2001 ; 18: 79-84$

6. Clyne B, Olshaker JS. The C-reactive protein. J Emerg Med 1999;17:1019-25.

7. Davies AH, Bernau F, Salisbury A, Souter RG. C-reactive protein in right iliac fossa pain. J R Coll Surg Edinb 1991;36:242-4.

8. Deohdare SG. C-reactive protein: clinical application. In: Johnson JM. Patho Articles. Update 2000-2001. Disponível em: URL: http://www.pathoindia.com.
9. Flum DR, Morris A, Koepsell T, Dellinger EP. Has misdiagnosis of appendicitis decreased over time? A population-based analysis. JAMA 2001;286:174853.

10. Fournier T, Medjoubi-NN, Porquet D. Alpha-1-acid glycoprotein. Biochim Biophys Acta 2000;1482:157-71.

11. Graffeo CS, Counselman FL. Appendicitis. Emerg Med Clin North America 1996;14:653-71.

12. Gurleyik E, Gurleyik G, Unalmiser S. Accuracy of serum C-reactive protein measurements in diagnosis of acute appendicitis compared with surgeon's clinical impression. Dis Colon Rectum 1995;38:1270-4.

13. Grönroos JM. Is there a role for leukocyte and CRP measurements in the diagnosis of acute appendicitis in the elderly? Maturitas 1999;31:255-8

14. Grönroos JM, Grönroos P. Leucocyte count and C-reactive protein in the diagnosis of acute appendicitis. Br J Surg 1999;86:501-4

15. Hallan S, Asberg A. The accuracy of C-reactive protein in diagnosing acute appendicitis - a meta-analysis. Scand J Clin Lab Invest 1997;57:373-80.

16. Lex A. Apendicites. In: Raia AA, Zerbini EJ, editores. Clínica cirúrgica Alípio Corrêa Netto. 4. ed. São Paulo: Sarvier; 1994. v.4, p.514-22.

17. Malcolm L, Brigden BL. Clinical utility of the erythrocyte sedimentation rate Am Fam Physian 1999;60:1443-50.

18. Mazlam MZ, Hodgson HJ. Why measure C reactive protein? Gut 1994;35:5-7.

19. Oosterhuis WP, Zwinderman AH, Teeuwen M, van Andel G, Oldenziel H, Kerkhoff JF, Siebbeles HW, van der Helm HJ. C reactive protein in the diagnosis of acute appendicitis. Eur J Surg 1993;159:115-9. 
Carvalho BR, Diogo-Filho A, Fernandes C, Barra CB. Leucograma, proteína C reativa, alfa-1 glicoproteína ácida e velocidade de hemossedimentação na apendicite aguda

20. Paajanen H, Mansikka A, Laato M, Kettunen J, Kostiainen S. Are serum inflammatory markers age dependent in acute appendicitis? J Am Coll Surg 1997; $184: 303-8$

21. Peltola H, Ahlqvist J, Rapola J, Rasanen J, Louhimo I, Saarinen M, Eskola J. Creactive protein compared with white blood cell count and erythrocyte sedimentation rate in the diagnosis of acute appendicitis in children. Acta Chir Scand 1986;152:55-8.

22. Rodriguez-San Juan JC, Martin-Parra JI, Garcia-Castillo L, Naranjo A. C-reactive protein and leukocyte count in the diagnosis of acute appendicitis in children. Dis Colon Rectum 1999;42:1325-29.

23. Sabiston DC Jr. Appendicitis. In: Sabiston DC Jr. Sabiston textbook of surgery. 15 ed. Philadelphia: Saunders; 1997. p.897-902.
24. Shakhatreh HS. The accuracy of C-reactive protein in the diagnosis of acute appendicitis compared with that of clinical diagnosis. Med Arh 2000;54:109-10.

25. Snyder BK, Hayden SR. Accuracy of leukocyte count in the diagnosis of acute appendicitis. Ann Emerg Med 1999;33:565-74

26. Steinman E. Apendicite aguda. In: Birolini D, Utyiama E, Steinman E, editores. Cirurgia de emergência. São Paulo: Atheneu; 1993. p.282-6.

27. Thimsen DA, Tong GK, Gruenberg JC. Prospective evaluation of C-reactive protein in patients suspected to have acute appendicitis. Am Surg $1989 ; 55: 466-8$

Recebido em 11/7/2002 Aprovado em 20/1/2003. 